Ann. Biol. anim. Bioch. Biophys., I96r, 1 (3), 31 I-316

\title{
NOTE PRÉLIMINAIRE SUR LES FACTEURS CONDITIONNANT LA TENEUR DE L'CEUF EN RIBOFLAVINE
}

\author{
J.-Cl. BLUM, Cl. CALET et Raymond JACQUOT \\ Avec la collaboration technique de Isabelle MACAIRE \\ Centre de Recherches sur la Nutrition, \\ Centre national de la Recherche scientifique, Bellevue (S. et O.), \\ et Station de Recherches avicoles, \\ (intre national de Recherches zootechniques, Jouy-en-Josas (S. et O.).
}

\section{SOMMAIRE}

Il peut exister de larges écarts dans la teneur en riboflavine d'œufs pondus par des poules de même race recevant une alimentation identique. On a étudié les facteurs pouvant être mis en cause :

La période de ponte ne joue pas en dehors du mois terminal.

La place de l'œuf dans la séquence de ponte n'intervient que faiblement et dans le seul cas des séquences longues.

La teneur du régime en riboflavine a une répercussion sur la teneur de l'œuf (vitellus et albumen): une surchage vitaminique conduit à un léger enrichissement du vitellus, une ration déficiente provoque un appauvrissement progressif de l'œuf entier et intensifie la chute normale observée dans le vitellus en fin de période de ponte.

La teneur en riboflavine de l'œuf est donc essentiellement fonction des aptitudes propres de chaque individu. Des analyses effectuées après ovariectomie partielle montrent la constance des teneurs en riboflavine de tous les ovocytes d'une même grappe, quelle que soit leur taille. Le transfert de la vitamine au vitellus constitue un processus continu et la teneur totale d'un ovocyte, blanc ou jaune, est fonction de sa masse et non de différences dans les teneurs relatives. La plus grande partie, sinon la totalité, de la riboflavine se dépose dans l'albumen au niveau du magnum sans qu'il y ait d'enrichissement ultérieur.

Plusieurs expérience ont été poursuivies pendant deux années pour préciser dans quelle mesure le taux de riboflavine du régime de la poule pondeuse retentit sur la teneur de l'œuf en cette vitamine. Les résultats initiaux montrent que cette teneur n'est pas constante chez des poules de race identique soumises à la même alimentation. Nous avons tenté de discriminer les facteurs responsables de ces écarts.

\section{MATÉRIEL, ET MÉTHODES}

Nous avons utilisé comme matériel expérimental des poules Rhode $\times$ Wyandotte choisies au cours de leur première année de ponte, d'après l'intensité de ponte des premiers mois, le poids moyen de l'œuf et le poids du corps. Les régimes sont complets et équilibrés, sauf en ce qui regarde le taux de riboflavine. Ils sont distribués ad libitum. 
Les œufs sont récoltés et conservés quelques jours au réfrigérateur en vue du dosage de la riboflavine dans le vitellus et l'albumen par la méthode microbiologique de SNELl et Strong, I939.

\section{RÉSULTATS E'T DISCUSSION}

Io Période de ponte.

Une expérience a été poursuivie de mars à juin, c'est-à-dire en pleine période de ponte ; le second essai a été fait de juin à fin août en fin de ponte. Si on suit la ponte individuelle de chaque poule, on voit qu'avec une ration équilibrée $(3,5 \mathrm{mg}$ de riboflavine $/ \mathrm{kg}$ ), la teneur' en riboflavine de l'œuf demeure pratiquement constante de mars à juin, puis diminue brutalement dès le début de juillet, l'appauvrissement portant essentiellement sur le vitellus.

Le graphique rend compte de ces observations.

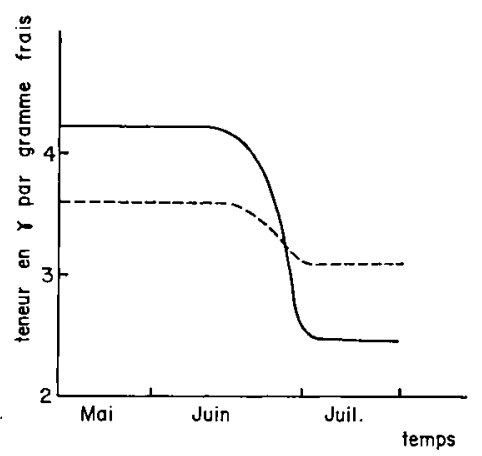

FIG. I. - Variation de la teneur en ribofla. vine, en fonction du mois de ponte (ration équilibrée $=3,5 \mathrm{mg}$ de riboflavine $\mathrm{kg}$ ) -. ouf entier -... vitellus.

$2^{\circ}$ Séquence de ponte.

Les résultats portant sur la composition en riboflavine du vitellus des oufs appartenant à des séries égales ou supérieures à 5 œufs figurent dans le tableau I. Nous n'y avons pas rapporté la composition des œufs des séquences plus courtes puisque dans ce cas la teneur en riboflavine des oufs est indépendante de l'ordre dans lequel ils sont pondus.

TABLEAU I

Influence de la place de l'ouf dans une séquence plus longue ou égale $\grave{a} 5$ jours sur la teneur en riboflavine du vitellus.

(régime normal : 3,5 $\mathrm{mg}$ de riboflavine $/ \mathrm{kg}$ d'aliment).

\begin{tabular}{|c|c|c|c|c|c|c|c|}
\hline \multirow{2}{*}{$n^{0}$ des poules } & \multicolumn{2}{|c|}{109} & \multicolumn{3}{|c|}{178} & \multirow{2}{*}{58} & \multirow{2}{*}{59} \\
\hline & $\begin{array}{c}1^{\mathrm{er}} \\
\text { exemple }\end{array}$ & $\begin{array}{c}2^{\mathrm{e}} \\
\text { exemple }\end{array}$ & $\begin{array}{c}1^{\mathrm{er}} \\
\text { exemple }\end{array}$ & $\stackrel{2^{\mathbf{e}}}{\text { exemple }}$ & $\begin{array}{c}3^{\mathbf{e}} \\
\text { exemple }\end{array}$ & & \\
\hline $\begin{array}{r}\text { Teneur moyenne en } \\
\mu g / g \text { dans les } 4 \\
\text { premiers oufs de } \\
\text { la séquence...... } \\
\text { Teneur moyenne en } \\
\mu g / g \text { dans les œufs } \\
\text { suivants ........ }\end{array}$ & 2,3 & 2,5 & 2,3 & 2,4 & 2,5 & 2,6 & 2,1 \\
\hline
\end{tabular}


Lorsque la séquence est longue on note une tendance à l'appauvrissement du vitellus des derniers œufs pondus. L'amplitude des écarts est cependant faible et ne s'observe qu'avec des rations pauvres en riboflavine.

Avec des régimes hypervitaminés, le facteur " séquence de ponte " ne joue plus. De son côté la teneur en riboflavine de l'albumen n'est en rien modifiée par la place de l'œuf dans la séquence.

$3^{\circ}$ Influence du régime.

Un régime déficient en vitamine $B_{2}(I, 85 \mathrm{mg} / \mathrm{kg})$ entraîne un appauvrissement lent et progressif de l'œuf en riboflavine, appauvrissement qui porte à la fois sur le vitellus et sur l'albumen et qui s'accentue en fin de ponte. Un régime hypervitaminé (125 $\mathrm{mg} / \mathrm{kg}$ ) conduit à un enrichissement du vitellus. Mais, comme l'avait déjà noté ADRIAN, I958, les variations sont de faible amplitude par rapport à la surcharge du régime.

Le tableau 2 rapporte les teneurs moyennes calculées sur la ponte de 3 poules.

TABL,EAU 2

Influence du régime hypervitaminé sur la teneur du vitellus

(I25 mg de riboflavine $/ \mathrm{kg}$ d'aliment).

\begin{tabular}{|c|c|c|}
\hline Date & Réggime & $\begin{array}{l}\text { Teneur en riboflavine du vitellus } \\
\qquad(\mu \mathrm{g} / \mathrm{g})\end{array}$ \\
\hline 10 mars -28 mars $\ldots \ldots \ldots \ldots \ldots$ & Normal & 2,4 \\
\hline 29 mars -5 avril $\ldots \ldots \ldots \ldots \ldots$ & Hypervitaminé & 3,7 \\
\hline 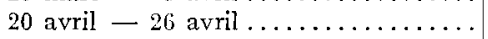 & - & 3,8 \\
\hline $4 \mathrm{mai}-10 \mathrm{mai} \ldots \ldots \ldots \ldots \ldots$ & - & 3,9 \\
\hline 17 juin -28 juin $\ldots \ldots \ldots \ldots \ldots$ & - & 2,9 \\
\hline
\end{tabular}

On voit en outre que la teneur du vitellus diminue en fin de ponte malgré le maintien du régime hypervitaminé.

\section{$4^{\circ}$ Infuence individuelle.}

Ce sont de loin les plus importantes. Avec un régime équilibré et pour le même mois de ponte (mai) les valeurs extrêmes d'individu à individu sont en $\mu \mathrm{g}$ par $g$ de 2,2 et 6,6 pour le vitellus, de $I, 6$ et 5,6 pour l'albumen, soit 2 et 5,2 pour le contenu total de l'œuf. Néanmoins, les valeurs extrêmes n'intéressent que quelques cas, ce qui justifie le calcul de moyennes. Par exemple, I9 valeurs sur 28 sont comprises entre 3 et $4 \mu \mathrm{g}$ de riboflavine par $\mathrm{g}$ de vitellus frais pour une moyenne de 3,7 ug calculée sur l'ensemble d'un lot'soumis au régime normal. En régime hypervitaminé, la marge des écarts individuels est du même ordre.

Il apparaît donc que chaque poule possède des aptitudes propres pour le transfert de la riboflavine à l'œuf.

\section{$5^{\circ}$ Etude de la grappe ovarienne.}

Il était dès lors intéressant d'étudier ce processus au niveau de la grappe ovarienne. Afin de doser les teneurs en riboflavine, on effectue une ovariectomie partielle et on prélève des ovocytes à divers stades d'évolution depuis l'ovocyte blanc d'un 
diamètre moyen de $6 \mathrm{~mm}$ jusqu'au jaune prêt à être ponđu. La ponte reprend quelques jours après l'opération. On récolte les œufs pour analyse. On peut répéter 1'intervention et la ponte reprenđra de la même façon.

En fin d'expérience toutes les poules sont sacrifiées et on dose la riboflavine dans les ovocytes restant sur les ovaires. Ce travail a porté sur 18 poules avec en moyenne I5 dosages par animal. Comme il est impossible ici de rendre compte de la totalité des résultats, nous ne rapporterons que quelques exemples représentatifs de l'ensemble (Tableau 3).

TABLEAU 3

Teneur en riboflavine ( $\mu \mathrm{g} / \mathrm{g}$ frais) des ovocytes en fonction de la taille.

\begin{tabular}{|c|c|c|c|c|c|c|c|}
\hline \multirow{2}{*}{$\begin{array}{c}\text { Diamètre } \\
\text { des } \\
\text { ovocytes } \\
\text { (en mm) }\end{array}$} & \multicolumn{2}{|c|}{$\begin{array}{l}\text { POULE } 109 \\
\text { (régime normal) }\end{array}$} & \multicolumn{2}{|c|}{$\begin{array}{c}\text { POULE } 178 \\
\text { (régime normal) }\end{array}$} & \multicolumn{2}{|c|}{$\begin{array}{l}\text { POULE 58 } \\
\text { (régime } \\
\text { hypervitaminé) }\end{array}$} & \multirow{2}{*}{$\begin{array}{c}\text { Moyennes } \\
\text { d'un lot } \\
\text { (régime } \\
\text { hypovitaminé) } \\
\text { ovariotomie }\end{array}$} \\
\hline & $\frac{2^{\mathrm{e}}}{\text { ovariotomie }}$ & sacrifice & $\frac{2^{\mathrm{e}}}{\text { ovariotomie }}$ & sacrifice & $\frac{2^{\mathbf{e}}}{\text { ovariotomie }}$ & sacrifice & \\
\hline moins de $8 \ldots$ & 1,5 & 2,0 & 3,0 & 2,7 & 4,5 & 3,2 & 1,2 \\
\hline $9-12 \ldots \ldots \ldots$ & 1,8 & - & 3,4 & 3,3 & 3,1 & - & 0,8 \\
\hline $13-17 \ldots \ldots \ldots$ & 1,5 & 1,5 & 3,2 & 2,4 & 3,2 & 2,5 & 2,0 \\
\hline $18-23 \ldots \ldots \ldots$ & 2,0 & 1,6 & 2,8 & 1,9 & - & 2,9 & - \\
\hline $24-28 \ldots \ldots \ldots$ & 1,8 & 1,7 & - & 2,3 & - & 2,9 & 2,1 \\
\hline Plus de $28 . \ldots$ & 1,8 & 1,8 & 3,1 & 2,3 & - & 2,7 & 1,9 \\
\hline Moyennes & 1,7 & 1,7 & 3,1 & 2,5 & 3,6 & 2,9 & 1,6 \\
\hline Jaune dans & & & & & & & \\
\hline l'oviducte. & & & & & & 3,2 & \\
\hline
\end{tabular}

On voit que :

- d'une poule à une autre, il existe des différences notables (du simple au double) dans la tenetrr en riboflavine des ovocytes bien qu'il s'agisse d'individus de la même race soumis au même régime (poules Iog et $\mathrm{I}_{7} 8$ ) ;

- par contre, ces teneurs varient peu dans une grappe ovarienne donnée quelle que soit la taille des ovocytes depuis le petit ovocyte blanc jusqu'au vitellus récolté dans l'oviducte.

L'institution d'une ration hypervitaminée cinq jours avant la seconde ovariotomie montre que l'influence du régime sur la teneur en riboflavine des ovocytes est immédiate avec tendance à la diminution ultérieure (Tableau 4).

TABLEAU 4

Teneur moyenne des ovocytes en riboflavine ( $\mu \mathrm{g} / \mathrm{g}$ frais)

en fonction des conditions alimentaires.

\begin{tabular}{c|c|c|c}
\hline \hline \multirow{2}{*}{$\mathrm{n}^{\text {o des poules }}$} & $\begin{array}{c}\text { Régime normal } \\
\text { 1 ere ovariotomie }\end{array}$ & \multicolumn{2}{|c}{ Régime hypervitaminé } \\
\cline { 3 - 4 } & 2,5 & 3,6 & sacrifice \\
\hline 58 & 2,3 & 3,6 & 2,9 \\
59 & 1,9 & 3,4 & 1,5 \\
\hline 215 & & & 1,9 \\
\hline
\end{tabular}


Signalons que cet effet se manifeste au niveau de tous les ovocytes indépendamment de leur taille.

\section{$6^{\circ}$ Albumen.}

On observe également une relation entre la teneur de l'albumen en riboflavine et celle du régime surtout lorsque ce dernier est déficient (Tableau 5).

TABLEAU 5

Teneur en riboflavine de l'albumen en fonction du régime.

\begin{tabular}{|c|c|c|c|}
\hline Régime & $\begin{array}{c}\text { Teneur du régime }(\mathrm{mg} / \mathrm{kg}) \\
\text { en riboflavine }\end{array}$ & Mois & $\begin{array}{l}\text { Teneur de l'albumen } \\
\text { en riboflavine }(\mu \mathrm{g} / \mathrm{g})\end{array}$ \\
\hline Déficient.............. & 1,85 & $\begin{array}{l}\text { juin } \\
\text { juillet }\end{array}$ & $\begin{array}{l}2,3 \\
0,7\end{array}$ \\
\hline Normal.... & $3,5-10$ & $\begin{array}{l}\text { juin } \\
\text { juillet }\end{array}$ & $\begin{array}{l}3,5 \\
3,4\end{array}$ \\
\hline
\end{tabular}

L'influence du régime déficient est progressive et dans le lot de poules qui le reçoivent on enregistre en fin de ponte un véritable effondrement des teneurs de l'albumen.

Par ailleurs, on trouve la même quantité totale de riboflavine dans l'albumen prélevé à la sortie du magnum et dans l'albumen de l'œuf pondu (Tableau 6.)

TABLEAU 6

Comparaison entre le contenu en riboflavine de l'albumen

à la sortie du magnum et dans l'œuf pondu.

\begin{tabular}{|c|c|c|c|c|}
\hline & \multicolumn{2}{|c|}{ Albumen à la sortie du magnum } & \multicolumn{2}{|c|}{$\begin{array}{l}\text { Albumen de l'ceuf pondu } \\
\text { (moyennes) }\end{array}$} \\
\hline & Poule 58 & Poule 59 & Poule 58 & Poule 59 \\
\hline $\begin{array}{l}\text { Poids }(g) \ldots \ldots \ldots \\
\text { Riboflavine }(; g) \ldots\end{array}$ & $\begin{array}{l}18 \\
98\end{array}$ & $\begin{array}{l}14,5 \\
145\end{array}$ & $\begin{array}{c}36 \\
99 \\
\text { (extrêmes } 163-60 \text { ) }\end{array}$ & $\begin{array}{c}37,7 \\
119 \\
\text { (extrêmes } 150-90 \text { ) }\end{array}$ \\
\hline
\end{tabular}

Ces résultats confirment les observations de BoLToN, I952. On peut en déduire que la plus grande partie, sinon la totalité, de la riboflavine se dépose dans l'albumen au niveau du magnum en même temps que les protéines sans qu'il y ait d'enrichissement ultérieur.

En résumé, la teneur en ribollavine de l'œuf est essentiellement réglée par un facteur intrinsèque caractérisant les aptitudes d'une poule donnée à transférer cette vitamine dans le vitellus et l'albumen. La période de ponte n'a que peu d'influence en dehors du mois terminal. La place de l'œuf dans la séquence de ponte n'intervient que faiblement et seulement pour des séquences longues. Le degré de vitaminisation du régime est un facteur de variation, mais d'amplitude restreinte. En effet, le pour- 
centage de riboflavine ingérée qui est transféré au vitellus varie en raison inverse des quantités ingérées. Le fait le plus intéressant est la constance des teneurs en riboflavine des ovocytes d'une grappe ovarienne donnée, quelle qu'en soit la valeur absolue. Ces teneurs sont celles que l'on retrouve dans le vitellus de l'œuf pondu. Le dépôt de riboflavine dans les ovocytes constitue un phénomène continu bien que les petits et les gros ovocytes diffèrent par la composition en eau, en lipides et en protéines. La quantité totale de ribollavine dépend donc essentiellement de la masse des ovocytes et non d'une modification des teneurs relatives.

Reçu en Févriè 1961

\section{SUMMARY}

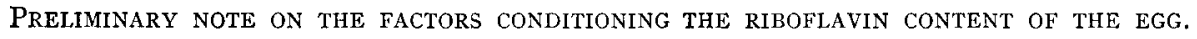

There may be great differences in the riboflavin content of eggs from hens of the same stock (Rhode Wyandotte in their first year of laying) which received the same complete and balanced diet. The factors probably responsible for this have been studied.

I) The laying period does not affect the riboflavin content of the vitellus, except for the last month. While the average content in the vitellus remains constant and superior to $4 \mu \mathrm{g}$ per $\mathrm{g}$ of fresh weight during May and June, it drops abruptly in July $(2,5 \mu \mathrm{g} / \mathrm{g}$.)

2) The position of the egg in the clutch plays but a slight part and only in the case of long clutches. On an average, the first four eggs contain an excess of $10 \mathrm{p}$. Ioo riboflavin in relation to the last eggs of the clutch.

3) The riboflavin content of the diet affects the content in the egg (vitellus and albumen), an extra amount resulting in a slight increase in the vitellus, a deficient diet leading to a decrease in the whole egg and intensifying the drop normally observed in the vitellus at the end of the laying period.

4) The riboflavin content of the egg is thus closely related to the natural disposition of each individual. Analyses carried out after partial ovariectomy show the constancy of the riboflavin content of all ovocytes of a same ovary, in the oocytes of a diameter lower than $8 \mathrm{~mm}$ (white follicles) as well as in the case of yellow follicles, whatever their size. The transfer of the vitamin to the vitellus is a continual process independent of the stage of development of the ovocytes, and their total content is related to their weight and not to the differences in their relative contents. The greater part, if not the whole of the riboflavin is deposited in the albumen together with the protein, at the time of the yolk passage through the magnum. No further increase is observed.

\section{RÉFÉRENCES BIBLIOGRAPHIQUES}

ADRIan J., 1958. Influence des surcharges vitaminiques du régime de la poule sur la teneur de l'œuf en certaines vitamines. Arch. Sci. Physiol., 12, ro7-140.

BOLTON W., 1952. Observations on the vitamin metabolism of the common fowl. 1. - The partition of free and combined riboflavin in the tissues of the laying hens. Brit. J. Nutr., 6, $7 \mathrm{I}-75$.

Snell E. E., Strong F. M., I939. A microbiological assay for riboflavin. Ind. Eng. Chem. Anal. Ed., 11, 346. 